\title{
Kontrolle, Legitimation, Reflexion
}

\section{Die Aufgaben der Selbstevaluation in der Jugendhilfe}

\author{
Birgit Kalter
}

Today, youth welfare has to proof what it does in which way and with whom, and with what kind of success. Supervision is the gateway between youth welfare and research. The cooperation of practitioners and researchers from outside can be fruitful for both sides.

Aujourd'hui, l'aide à la Jeunesse doit prouver ce qu'elle fait, comment, avec qui et avec quel succès. $\mathrm{La}$ supervision est la voie entre l'aide à la jeunesse et la recherche. La coopération entre praticiens et chercheurs de l'extérieur peut être très fructueuse pour les deux parties.

Birgit Kalter ist wissenschaftliche Mitarbeiterin des Instituts für Stadtteilbezogene Soziale Arbeit und Beratung (ISSAB) der Universität Duisburg-Essen.

E-Mail birgit.kalter@t-online.de
Die Jugendhilfe muss heute nachweisen, was sie wie mit wem und mit welchem Erfolg tut. Die Schnittstelle von Jugendhilfe und Forschung bildet die Selbstevaluation. Dabei ist die Zusammenarbeit der Praxis mit externen Forschern durchaus nützlich für beide Seiten.

Fragt man nach der Schnittstelle von Jugendhilfe und Forschung, so lautet die naheliegende Antwort "Jugendhilfeforschung «. Einerseits zutreffend, muss man sich andererseits der für beide Seiten bitteren Wahrheit stellen, dass Forschungsbefunde - so nachvollziehbar und wertvoll sie auch immer sein mögen - von der Praxis nicht unmittelbar aufgegriffen und als handlungsrelevant akzeptiert werden. Hierfür spricht beispielsweise, dass mit den Studien "Was leistet Heimerziehung? « (Planungsgruppe Petra 1985), »Leistungen und Grenzen der Heimerziehung « (JULE) unter der Leitung von Hans Thiersch (1999) und »Effekte erzieherischer Hilfen und ihrer Hintergründe « (JES) (2002) unter der wissenschaftlichen Beratung von Martin Schmidt und Franz Petermann Forschungsprojekte öffentlich beauftragt, finanziert und innerhalb der Jugendhilfe realisiert sowie umfangreich und kostenlos veröffentlicht, aber diese kaum nachvollziehbar rezipiert wurden. Entsprechend lässt sich Jugendhilfeforschung im Großen und Ganzen als Schnittstelle von Jugendhilfe und Forschung nur bedingt ausmachen: Sie bildet zwar eine Verbindungsstelle zwischen den beiden Systemen, bleibt aber als Programm, das die Kommunikation zwischen den beiden Funktionsgruppen unterstützt, zwischen ihnen vermittelt oder sie gar wirkungsvoll miteinander verbindet, relativ blass.

Anders verhält es sich, wenn Jugendhilfeeinrichtungen sich aus eigenem Erkenntnisinteresse Forschung nähert: Jenseits von Debatten um das Verhältnis von Forschung und Praxis etabliert sich gerade im Zusammenhang mit sozial- raumorientierten Aktivitäten eine Wirklichkeit zahlreicher Evaluationsfragen und Evaluationsprojekte. Mit der Durchführung systematischer Beschreibungen und Bewertungen (von Ausschnitten) des eigenen Handelns und seiner Auswirkungen nach selbst bestimmten Kriterien (vgl. König 2000, 38) setzt sich hier eine lange Tradition von Selbstevaluation fort, die aktuell das $\mathrm{Zu}$ sammenwirken zwischen Jugendhilfe und Forschung verändernd zu akzentuieren scheint.

\section{Sozialräumlich orientierte Jugendhilfe}

Selbst wenn man vermuten darf, dass die Motive und Zielsetzungen durchaus unterschiedlich gewichtet sind, bleibt es Tatsache, dass bundesweit derzeit zahlreiche Städte und Gemeinden daran arbeiten eine sozialraumorientierte Jugendhilfe aufzubauen. Mittlerweile dem Experimentier- und Modellstatus entwachsen, wird vielerorts zwischen dem öffentlichen Träger und den freien Trägern der Jugendhilfe ein gemeinsames sozialräumliches Arbeiten vereinbart, das sich auf das Fachkonzept Sozialraumorientierung stützt und mit einem anspruchsvollen Umgestaltungsprozess verbunden ist.

Dessen Spektrum reicht von der Neujustierung der Aufgaben und Zugangsweisen des Allgemeinen Sozialen Dienstes »über neue Formen der Kooperation zwischen Kostenträger und Leistungserbringern im Bereich der Hilfen zur Erziehung und - damit einhergehend - einem gesteuerten, inhaltlich geprägten Umbau der lokalen Trägerlandschaft bis hin zu innovativen Formen der Gestaltung von strukturstützenden Verfahren etwa im Bereich der Hilfeplanung, der Leistungsund Entgeltvereinbarung sowie der besseren Kooperation zwischen verschiedenen Leistungsfeldern der Jugendhilfe « (Hinte 2006, 19). 
Orientierung und Richtschnur für den ortsspezifischen Gesamtprozess und für das auf eine Gebietseinheit bezogene Agieren der Fachkräfte bilden - bei aller Individualität in der Erledigung der jeweils konkreten Aufgaben - die mit dem Fachkonzept verbundenen Prinzipien (ausführlich hierzu Hinte/Treeß 2007): Die Orientierung am Willen und den Interessen der hilfesuchenden Menschen; die grundsätzlich aktivierende, Ressourcen des Quartiers und der dort lebenden Menschen aufgreifende Ausrichtung; die zielgruppen- und bereichsübergreifende und Kooperation und Vernetzung suchende Herangehensweise bilden jene Maßstäbe, unter denen Teilaspekte des Umbauprozesses analog analysiert, bearbeitet und evaluiert werden. In Verbindung gebracht mit strategischen Zielen fließen sie in Qualitätsentwicklungs- und Controllingvereinbarungen ein. Diese bilden Grundlage für Systeme der Selbstevaluation, mit deren Hilfe der gemeinsame Prozess von Jugendamt und freien Trägern abgesichert und verfolgt und sowohl fach- wie kommunalpolitisch für ausreichend Transparenz gesorgt werden soll.

\section{Selbstevaluation sozialraum- orientierter Jugendhilfe}

Dass es sich bei den selbstevaluierenden Aktivitäten sozialräumlich orientierter Jugendhilfe nicht um etwas grundlegend Neues handelt, lässt sich durch zahlreiche Beispiele belegen. Eine beeindruckende Liste solcher frühen Evaluationsvorhaben findet sich beispielsweise in einem Beitrag von Hermann Stutte über »Methodik und Ergebnisse der Bewährungsprüfungen bei ehemaligen Fürsorgezöglingen « im Handbuch der Heimerziehung von 1952 (dazu näher Wisskirchen/Kibben 1985). Schon den Pionieren Sozialer Arbeit ging es darum, mit Blick auf die Möglichkeiten der Sozialpädagogik sowohl Begründungen für ihr jeweiliges Konzept und Vorgehen zu finden, als auch Fragen nach der Umsetzung und Zielerreichung zu beantworten.

Der Blick in die Geschichte zeigt aber auch Intentions- und Anforderungsverschiebung: Während es in Zeiten des wirtschaftlichen Aufschwungs programmatisch als Legitimation auszureichen schien, dass für sozialpolitisch anerkannte Problemlagen überhaupt etwas getan wurde, so hat sich diese Sichtweise mit dem wachsenden gesellschaftspolitischen Druck auf die vor allem aus öffentlichen Mitteln finanzierten sozialen Aufgaben deutlich verändert (vgl. Kalter/ Schneider 2004, 93): Parallel zu Sparzwängen und Kostendruck ist die Forderung gewachsen, dass »soziale Arbeit sich legitimiert für das, was sie tut, wie sie es tut, mit welchem Erfolg und mit welchem Klientel« (BMFSFJ 1999, 313).

Vor dem Hintergrund der Qualitätsdebatte und der Debatte um "Neue Steuerung « haben Selbstevaluationen Ende des letzten Jahrhunderts auch in der Jugendhilfelandschaft einen Aufschwung erlebt. definierter Ziele, sondern vor allem über Wirkmechanismen in Kenntnis zu setzen, um auf dieser Basis zu einer verantwortlichen, professionellen, kritisch-reflektierenden Prüfung der Angemessenheit von Interventionen $\mathrm{zu}$ gelangen. (vgl. Otto 2007, 54)

Vor diesem Hintergrund betrachtet zeigt sich, dass die mit sozialraumorientierter Jugendhilfe verbundenen Evaluationen einerseits durchaus zur Kontrolle und Legitimation genutzt werden: Geprüft wird, ob und inwieweit die mit den Umbauprozessen verbundenen Strukturund Prozessmerkmale überhaupt realisiert und intendierte Ziele erreicht wer-

\section{»Evaluation kann nicht aufoktroyiert, sondern muss ausgehandelt werden «}

Das "Zauberwort Evaluation" hatte zwar zwischenzeitlich "etwas von seiner Strahlkraft eingebüßt « (Bröckling 2004, 76), scheint mit der bundesweit geführten »Wirkungsdebatte « jedoch erneut zu erstarken (hierzu aktuell IGFH 2006 und www.wirkungsorientierte-jugendhilfe.de). Selbstevaluation in der Jugendhilfe - so könnte man den aktuellen Stand $\mathrm{zu}^{-}$ sammenfassen - bewegt sich zwischen den Intentionen einerseits Steuerungsparameter zu erhalten und ihre Leistungen und Ergebnisse offen zu legen. Andererseits sollen die Befunde zur Qualifizierung der eigenen Arbeit genutzt werden. Evaluationsprojekte in der Jugendhilfe ausschließlich mit Steuerungsfantasien in Verbindung zu bringen, schiene zumindest dann bedenklich, » wenn man glaubt oder suggeriert, dies bedeute, man könne dieses komplexe Feld zielgerichtet systematisch so beeinflussen, dass man es irgendwie >in den Griff $<$ bekommt « (Hinte 2006, 8). Ebenso deutlich bliebe Selbstevaluation hinter ihren Möglichkeiten zurück, würden deren Verwertungszusammenhänge auf Kosten-Nutzen-Analysen und auf Fragen der Vergleichbarkeit von Effizienz und Leistungen reduziert. Sinnvoll erscheint demnach, sich mittels Forschung und Selbstevaluation nicht nur über relative Erfolgswahrscheinlichkeiten von Maßnahmen hinsichtlich spezifisch den. Hier durchaus neu ist hingegen, dass sich die systematischen Beobachtungen, Dokumentationen und Analysen weder auf kleinteilige Segmente der Jugendhilfe wie etwa Hilfepläne, noch auf Spezialleistungen wie Hilfen der Erziehung, noch auf Einzelinstitutionen oder Träger beschränken: Einzelsegmente, -leistungen und -institutionen werden allenfalls im Zusammenhang und im Dienste des Gesamtprozesses evaluiert. Indem der »Sozialraum « als Steuerungsgröße in den Vordergrund gestellt wird, werden auch bedarfs-, gestaltungs- und ergebnisbezogene Parameter - und diese institutionsübergreifend - entwickelt und die von der inhaltlichen Konzeption geprägten Vorgehensweisen in Bezug auf alle Bereiche der kommunalen Jugendhilfe und auf Chancen des wechselseitigen Bezugs bewerten (ausführlich Hinte 2006, 8 f.).

Evaluationsgegenstände, -indikatoren und -kriterien werden nicht von einer Seite aufoktroyiert, sondern gemeinsam ausgehandelt und Bedingungen, Methoden und Durchführungsfragen werden gemeinsam geklärt. Somit werden Selbstevaluationen von vorneherein auf »breitere Füße " gestellt und Befunde in einen Regelkreis gemeinsamer Qualitätsentwikklung eingebracht. Entsprechend erhöht sich die Wahrscheinlichkeit institutionsübergreifender Reflextionsprozesse. Und: 
Dort, wo Evaluationsergebnisse und statistische Zusammenhänge nicht einfach als Wenn-dann-Kausalitäten abgebildet, sondern in Bezug auf wahrscheinliche oder plausible Wirkungszusammenhänge einer näheren Betrachtung und fachlichen Interpretation unterzogen werden, bieten sie gute Chancen zur Stärkung reflexiver Professionalität und zur fachlichen Weiterentwicklung lokaler Jugendhilfesysteme beizutragen.

\section{Schnittstelle von Jugendhilfe und Forschung}

Die Selbstevaluationsprozesse sozialraumorientierter Jugendhilfe sind demnach ebenso anspruchsvoll wie deren Etablierungs- und Arbeitsweisen: Sich auf ein gemeinsames Qualitätsentwicklungs- und Controllingsystem zwischen öffentlichem und freien Trägern zu einigen, einen diesbezüglichen Kontrakt zu erarbeiten, gemeinsame strategische Ziele und fachlich inhaltliche Standards festzulegen, Indikatoren und Kennzahlen sowie alltagsintegrierbare Überprüfungsmethoden und -instrumente zu entwickeln und einzuführen, wiederkehrend Ergebnisse auszuwerten, aufbereitet zu repräsentieren und dem fortlaufenden Prozess gemeinsamer Interpretation und Entwicklung zur Verfügung zu stellen, setzt Kenntnisse und Erfahrung ebenso voraus wie verfügbare Ressourcen.

Dass Selbstevaluation kaum neben dem Alltagsgeschäft leistbar ist, bildet nicht den einzigen Grund, die Dienste sozialpädagogischer Forschungs- und Beratungsinstitutionen in Anspruch zu nehmen. In Abhängigkeit von den Gegebenheiten vor Ort werden externe Unterstützungen in unterschiedlicher Form und Tiefe gesucht: von evaluationsbezogenen Weiterbildungen, über Beratung bei der Erstellung eines Selbstevaluationskonzepts, über Begleitung und Unterstützung der Evaluationsprozesse bis hin zur externen Gesamtplanung, -durchführung, -auswertung und Dokumentation.

Die sozialpädagogische Orientierung der Institute gewährleistet in der Regel, dass sich deren Forschungszugang nicht nur auf die Gegenstände der Sozialpädagogik bezieht, sondern sie sich auch methodisch und in den relevanten Theoriebezügen als spezifisch sozialpädagogisch ausweisen. Kurz gesagt, man spricht eine gemeinsame Sprache und stellt sicher, dass die Selbstevaluationsprozesse sich auf sozialpädagogische Theorie stützen und beziehen und aus dem Repertoire der Forschungsmethoden jene kombiniert werden, die zur Beantwortung der Fragestellung und zur Auseinandersetzung mit dem was Sozialpädagogik und Jugendhilfe leisten, am ehesten geeignet scheinen.

Eine externe Instanz zu beteiligen und mit einem entsprechenden Auftrag auszustatten, unterstützt die Priorität, die durchaus unterschiedlichen Einrichtungseinzelinteressen in das gemeinsame Ziel zu integrieren. Es unterstützt zudem, dass das Evaluationsinteresse in seinen Zielen, methodischen Zugängen und Auswertungen immer wieder kritisch an den fachlich inhaltlichen Positionen der Sozialraumorientierung geprüft und auf sie zurückgebunden wird. Auf dem Fundament (Prinzipien) des Konzepts stehend, regen die Selbstevaluationsprozesse die Suche nach eigenen ortsspezifischen Gestaltungsformen an.

Wie lebendig hieraus Anpassungsleistungen, Stilwechsel und Darstellungsvarianten entstehen, ohne dass der grundlegende Charakter des Gebäudes "Sozialraumorientierung " dabei verloren geht (vgl. Hinte 2006, 9), wird durch eine Vielzahl aktueller Veröffentlichungen belegt (Hinte/Treeß 2007; Budde/ Früchtel/Hinte 2006; Kalter/Schrapper 2006). Die hier dargelegten Beschreibungen, Analysen und Bewertungen verweisen auf ein Zusammenwirken von forschungsinteressierten Praktikern und praxisinteressierten Forschern, das sich nicht nur für beide Seiten lohnt und beiden Seiten Anregung bietet, sondern auch zweckdienlich ist sowohl zwischen den beiden Systemen zu vermitteln als auch etwas Gemeinsames sowie etwas gemeinsam Neues entstehen zu lassen.

\section{Literatur}

Bröckling, U. (2004): Evaluation. In: Bröckling, U./Krasmann, S./Lemke, T. (Hg.): Glossar der Gegenwart. Frankfurt am Main, S. 76-81.

Budde, W./Früchtel, F./Hinte, W. (Hrsg.) (2006): Sozialraumorientierung. Wege zu einer veränderten Praxis. Wiesbaden.

BMFSFJ (Hg.) (1999): Handbuch Sozialpädagogische Familienhilfe. Bonn.

Hinte, W./Treeß, H. (2007): Sozialraumorientierung in der Jugendhilfe. Theoretische Grundlagen, Handlungsprinzipien und Praxisbeispiele einer kooperativ-integrativen Pädagogik. Weinheim, München.

Hinte, W. (2006): Geschichte, Quellen und Prinzipien des Fachkonzepts »Sozialraumorientierung «. In: Budde, W./Früchtel, F./Hinte, W. (Hg.): Sozialraumorientierung. Wege zu einer veränderten Praxis. Wiesbaden. S. 7-21.

Internationale Gesellschaft für erzieherische Hilfen (IGFH) (2006): Forum Erziehungshilfen. Thema: Wirkungsorientierung. 5/2006.

Kalter, B./Schrapper, C. (Hg.): Was leistet Sozialraumorientierung. Konzepte und Effekte wirksamer Kinder- und Jugendhilfe. Weinheim, München.

Kalter, B./Schneider, V. (2004): Praxisprüfung und Theoriebildung. Evaluation als anwendungsorientierte Sozialforschung. In: Schrapper, C. (Hg.): Sozialpädagogische Forschungspraxis. Positionen, Projekte, Perspektiven. Weinheim, München. S. 91100.

König, J. (2000): Einführung in die Selbstevaluation. Ein Leitfaden zur Bewertung der Praxis Sozialer Arbeit. Freiburg im Breisgau.

Stutte H. (1952): Methodik und Ergebnisse der Bewährungsprüfungen bei ehemaligen Fürsorgezöglingen. In: Trost, F. (Hg.): Handbuch der Heimerziehung. Bd. 1. Frankfurt am Main. S. 560-564.

Otto, H.-U. (2007): Zum aktuellen Diskurs um Ergebnisse und Wirkungen im Feld der Sozialpädagogik und Sozialarbeit -Literaturvergleich nationaler und internationaler Diskussion. Bielefeld.

Wisskirchen, M./Kibben, S. (1985): Erfolge der Fürsorgeerziehung. Untersuchungen zur »Lebensbewältigung «. In: Schrapper, C./Sengling, D.: Waisenhäuser und Erziehungsanstalten in Westfalen. Werkstattberichte zur Wanderausstellung »Geschichte der Kinder- und Jugendfürsorge«. Münster. S. 157-166. 